\title{
Sylvie Kandé, le « texte métis » de la poésie
}

\section{Dominique Combe}

\section{(2) OpenEdition}

\section{Journals}

\section{Édition électronique}

URL : http://journals.openedition.org/edl/2476

DOI : $10.4000 /$ edl. 2476

ISSN : 2296-5084

\section{Éditeur}

Université de Lausanne

\section{Édition imprimée}

Date de publication : 15 décembre 2017

Pagination : $15-30$

ISBN : 978-2-940331-66-6

ISSN : 0014-2026

\section{Référence électronique}

Dominique Combe, «Sylvie Kandé, le « texte métis » de la poésie », Études de lettres [En ligne], 3-4 |

2017, mis en ligne le 15 décembre 2019, consulté le 17 décembre 2020. URL : http://

journals.openedition.org/edl/2476; DOI : https://doi.org/10.4000/edl.2476

(c) Études de lettres 


\section{SYLVIE KANDÉ, LE «TEXTE MÉTIS» DE LA POÉSIE}

L'hybridité, chez Sylvie Kandé, désigne autant la matière que la forme de ses recueils. Cet article éclaire les différentes significations que cette notion prend pour la poète: celle de la diversité de voix convoquées et mêlées (poètes, artistes, historiens et penseurs d'horizons différents) ; celle du métissage des corps dans Lagon, lagunes; ou celle des gestes quotidiens liés à d'autres mouvements - Gestuaire - appartenant au temps de la colonisation. Entre les continents et les identités, la poésie de Sylvie Kandé navigue telle une pirogue, symbole majeur dans La quête infinie de l'autre rive, épopée en trois chants.

Sylvie Kandé entretient un dialogue intime et profond avec les poètes, les peintres, les musiciens, les historiens ${ }^{1}$, les penseurs d'Europe, d'Afrique et des Amériques, d'une rive à l'autre de «l'Atlantique noir». Dans le «maelström» de la mémoire individuelle et collective ${ }^{2}$, elle dédie ses deux premiers livres de poésie, Lagon, lagunes - tableau de mémoire (2000) accompagné d'une postface d'Édouard Glissant, à la romancière Marie Ndiaye et La quête infinie de l'autre rive, épopée en trois chants (2011), à l'historien Joseph Ki-Zerbo et tisse ainsi les liens de la Relation entre l'Afrique et le monde. Pour chaque poème, elle s'appuie sur des citations en épigraphe de Césaire, de Glissant aussi bien que de Pessoa ou d'Artaud. Tissés de nombreuses allusions ou références historiques, artistiques ou littéraires à l'Afrique et aux "mondes noirs",

I. Littéraire de formation, Sylvie Kandé est l'auteure d'une thèse soutenue à l'Université Paris VII en 1992 et intitulée Terres, urbanisme et architecture "créoles» en Sierra Leone, XVIII ${ }^{e}-X I X^{e}$ siècles.

2. É. Glissant, "Aux orées des archipels", postface à Lagon, lagunes - tableau de mémoire, p. 76. 
recensées et explicitées en fin de volume, comme dans Lagon, lagunes, les recueils font entendre des voix multiples, entrecroisées et métissées. Léopold Sédar Senghor et Birago Diop, venus d'Afrique, Léon-Gontran Damas et Aimé Césaire de la Guyane et des Antilles, Langston Hughes, W. E. B. Du Bois et Faulkner des États-Unis, répondent à Aloysius Bertrand, Baudelaire, Victor Segalen et Francis Ponge, en métropole. Les paysages, inspirés des tableaux de Gauguin, d'Alice Martinez-Richter ou du peintre haïtien Édouard Duval-Carrié, se devinent en écho des voix de Cassandra Wilson et de Joe Cocker, ou de celle du saxophone de John Coltrane.

De ce Récit-Relation et des échanges transatlantiques témoigne encore son recueil Gestuaire, publié en 2016, qui se fonde sur les récits d'esclaves recueillis aux États-Unis, à la fin des années trente, dans les États $\mathrm{du} \mathrm{Sud}^{3}$. Les poèmes "Cannes», "Coup de chapeau» ou «Au sujet du retable des neuf esclaves", d'après le tableau du peintre haïtien Édouard Duval-Carrié, mettent en scène l'univers de la plantation commun à Césaire et à Glissant.

C'est ainsi que, au plan phonétique aussi bien que sémantique, Gestuaire fait écho à Moi, laminaire, le dernier recueil publié par Aimé Césaire en 1982, dont le titre désigne une algue de la mer des Caraïbes. Césaire y exprime son attachement au "pays natal» et au "calendrier lagunaire», sur lequel il ouvre son recueil:

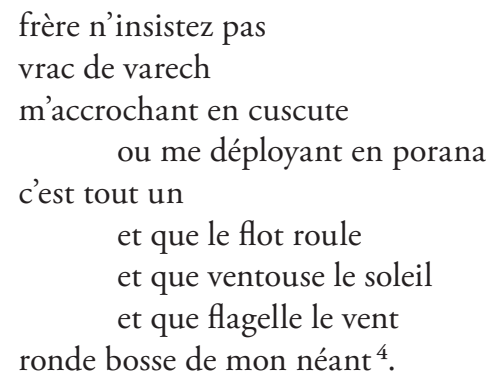

3. Born in slavery: slave narratives from the federal writers' project, 1936-1938.

4. A. Césaire, Moi, laminaire, p. 98. 
Dans le poème d'ouverture à Gestuaire, Sylvie Kandé évoque à son tour de manière toute césairienne "ces rochers gluants où l'algue mousse / Tant de secrets saumâtres hantent les marées basses!» (p. 11). Le nom même de Césaire apparaît d'ailleurs dans la dédicace du poème "Cannes» (p. 14), aux côtés de celui d'Édouard Glissant et de SaintJohn Perse, "clairvoyants visités par l'obscur". Quant à son premier recueil publié en 2000, Lagon, lagunes - tableau de mémoire, il affiche son hybridité générique, à la manière du Cahier d'un retour au pays natal. Composé de vers libres, de poèmes en prose et de proses poétiques, de contes, de fragments dramatiques ou de scènes de théâtre, il est sous-titré «tableau de mémoire». Cette œuvre polyphonique n'est pas pour autant un recueil disparate, du fait de son unité thématique, placée sous les auspices de Michel Leiris, cité en exergue: «L'Afrique - qui fit - refit et qui fera». Sylvie Kandé fait des paysages d'Afrique et des Antilles le «vrai lieu» pour une poésie du lagon, de la lagune et de la mangrove, où pousse justement l'algue laminaire. Dédié à Marie Ndiaye, postfacé par Édouard Glissant, le «tableau de mémoire» de Lagons, lagunes relie étroitement les Antilles à l'Afrique par une histoire de la Traite inscrite dans la chair comme dans le paysage, des terres rouges d'Ibadan jusqu'à la Montagne Pelée. La vie du mulâtre Vincent Ogé, martyr à SaintDomingue, et celle de Toussaint Louverture y sont mises en perspective avec celle de l'empereur du Mali Soundiata; telles des échos, ces traces d'humanité fondent ce que Glissant appelle un «texte métis», "qui nous porte au bord de ce qui nous tente: l'emmêlement, la saoulerie de n'avoir plus à exclure, oui, enfin, ce vertige-là ». Une griserie qui associe plus qu'elle ne sépare, passant par-delà la distinction des genres littéraires qui, «nous le savons déjà, s’emmeurent» (p. 76).

Dans Lagon, lagunes, Césaire fait l'objet de trois citations extraites du Cahier d'un retour au pays natal et de Et les chiens se taisaient. Ainsi, la figure de la Rebelle, avec qui l'empereur s'entretient, évoque directement le héros prométhéen de la tragédie Et les chiens se taisaient, publiée d'abord sous la forme d'un poème dramatique - «oratorio lyrique», écrit Césaire - dans Les armes miraculeuses, en 1946. Le paysage lagunaire du dernier poème du recueil de Sylvie Kandé, en vers libres celui-là, est proche de Moi, laminaire jusque dans la somptueuse richesse lexicale, toute césairienne, de registre savant: 
De grasses huîtres brunes moussaient dans la torpeur des mangroves. Au bord de l'éclampsie,

La lune pend comme un fruit mûr balancé aux branches du ciel.

La sueur ennoie jusqu'à la fuscine de sa pupille $[\ldots]^{5}$

À la «torpeur des mornes" dans le poème de Césaire intitulé «Mangrove», à la «torpeur de l'histoire» du poème éponyme, répond ici la «torpeur des mangroves».

Prolongeant les fils de l'intertextualité dans ce même recueil, Sylvie Kandé reprend à son compte la réponse célèbre de Senghor - dans la postface à Éthiopiques - à la question «Pourquoi écrivez-vous en français?»:

Parce que nous sommes des métis culturels, parce que nous sentons en nègres, nous nous exprimons en français, parce que le français est une langue à vocation universelle, que notre message s'adresse aussi aux Français de France et aux autres hommes $[\ldots]^{6}$

C'est ainsi que l'hybridité qui définit la matière aussi bien que la forme de Lagon, lagunes devient l'emblème de la poésie elle-même, de toute poésie:

nous les poètes

nous sommes les mulâtresses de plantation

nous désirons le maître et nous désirons

et les mots semés sur la page sont nos grains de beauté, nos envies, nos taches de son, de vin ou plutôt de café7.

\section{"Mulâtresse», "toubabesse»}

Pourtant, la Relation entre l'Afrique, l'Europe et les Amériques, indissociable du commerce triangulaire, n'est pas seulement culturelle, elle procède du corps. Le métissage est d'abord celui du «Sang noir», auquel Glissant a dédié son premier recueil de poèmes. Sylvie Kandé assume,

5. S. Kandé, Lagon, lagunes - tableau de mémoire, p. 61.

6. L. S. Senghor, Poèmes, p. 166.

7. S. Kandé, Lagon, lagunes - tableau de mémoire, p. 56. 
revendique même la pluralité d'une identité métisse de "sang-mêlé»" Plus encore que la couleur de peau et le corps lui-même, c'est le nom propre, le nom du père qui trahit l'infamie du métissage. Lors de la distribution des prix relatée dans Lagon, lagunes, c'est «l'appel de ce nom hors raison alphabétique, un barbarisme incongru doublé d'une sorte de traîtrise» (p. 16) qui suscite la réprobation des parents et la honte de qui s'entend dire: "certaines alliances sont condamnées à ne produire que d'extravagants bicéphales» (p. 17). Ainsi, c'est un double rejet que l'enfant subit, de part et d'autre - comme mulâtresse et comme toubabesse, tout à la fois. Humiliée par un professeur blanc, qui ne peut croire que le nom de son père soit celui d'un chimiste, la fillette est en bute au préjugé raciste le plus brutal, dont la violence est encore soulignée par l'absence de ponctuation:

Quelle fatuité que la vôtre (siffla la petite C. lisant par-dessus mon épaule) je tiens de mes parents que votre père est un balayeur moimême je l'ai vu hier dessus la promenade donner à votre frère l'argent pour son goûter 9 .

Les injures stéréotypées à caractère raciste fusent dans la «banlieue grise» (p. 36):

Rentre dans ton pays rentre chez toi rien à faire que les tiens ont toujours bien mérité de la France croix de guerre palmes académiques et tout le tralala t'auras beau montrer tes papiers pas d'erreur ta carte beige c'est ton mufle il nous revient pas dégage et plus vite que ça la patrie ça ne se quémande pas ${ }^{10}$.

Mais, réciproquement, les invectives n'empêchent pas, bien au contraire, l'exclusion en retour quand, sur le marché, à Dakar, la marchande "pointe du doigt dans [s]a direction":

- ...... Toubabesse... (j'entends au milieu des vrilles de son rire) Je me retourne pour voir qui, pour rire moi aussi de la badaude sanglée dans un tailleur safari ou embabouchée de jaune safran : à Dakar, avec les touristes, c'est selon.

8. Sylvie Kandé a dirigé et publié un colloque sur le métissage à New York University en 1997: Discours sur le métissage, identités métisses. En quête d'Ariel.

9. S. Kandé, Lagon, lagunes - tableau de mémoire, p. 35.

Io. Ibid., p. 36. 
Mais dans ce coin du marché, personne d'autre que moi à qui le geste s'adresse ${ }^{11}$.

Né d'une alliance contre nature, stigmatisé par «sa différence» ${ }^{12}$ et inassignable à une identité univoque, l'enfant métis est voué à une double exclusion - out of place, dirait Edward Saïd. Comme l'observe le poète et essayiste haïtien Joël Des Rosiers, Métis, fils de Zeus et d'Ouranos, a été assassiné par son père pour lui avoir volé la connaissance:

Cet infanticide est la punition réservée à toutes les figures de l'instable, de l'interlope, de l'hybride ${ }^{13}$.

Marquée par la lecture de Peau noire, masques blancs de Frantz Fanon, Sylvie Kandé décrit le choc des mots, qui déclenche la prise de conscience. Le traumatisme produit par le mot mulâtresse, jeté littéralement à la figure de l'enfant comme un "seau de sable», fait brutalement tomber les «écailles» de ses yeux, selon la formule biblique. Comme le montrent les premières pages de Lagon, lagunes, c'est de cette humiliation originelle que naît l'écriture:

Brusquement,

une comptine que chantaient autrefois les enfants du square à l'approche de la petite fille au visage lunaire, et où revenait un nom inconnu mais burlesque,

si burlesque vraiment, qu'en le retrouvant sous une pile de souvenirs, au bas du meuble près des livres, j'ai bien failli en rire.

Autrefois, elle en restait interdite, la petite, bouche bée et bras mous. Jusqu'au jour où, animé par la chanson, un des enfants lui jeta un seau de sable bien tassé en prévision d'un pâté. C'est avec un papier fin, un papier-cigarette je crois, que ma mère $\mathrm{a}$, des heures durant, retiré de mes yeux les écailles de sable.

- À présent je vois (me suis-je alors dit).

D'abord ces mains de femme dont je connais par cœur le paysage.

Au bout de la table l'effigie d'un coq en ergots sur le couvercle d'une boîte ordinaire.

II. Ibid.

I2. Titre d'une chanson et d'un album de Salif Keita paru en 2009.

I3. J. Des Rosiers, Théories caraïbes, poétique du déracinement, p. 188. 
Les draps blancs là-bas qui claquent aux balcons de la cité. Tant d'autres choses. Au fond, il ne reste qu'une question, là, plus-quelancinante - ce nom burlesque qu'on me donne et que j'ignore mulâtresse

Ma mère, qui connaissait tous les mots du dictionnaire, m’a fixée un instant, puis a balayé ma question et les derniers grains de sable.

Le temps s'égouttait sans bruit.

Une lune rousse comme un avril s'est enfin assise au zénith, et vague après vague, je m'élançais vers le sein replet de la très féconde, au risque de m'y glacer ${ }^{14}$.

C'est au retournement de "mulâtresse», mot vieilli et «burlesque», qu'est donc vouée la poésie, destinée à panser la blessure et à réparer l'humiliation.

Sylvie Kandé, en ce sens, procède de la même manière qu'Aimé Césaire avec le mot "nègre" dans le Cahier d'un retour au pays natal. En écho à la «négraille» du Cahier, Jean-Baptiste Chavannes, lui-même métis, envoie "à la canne, la mulâtraille" des "sang-mêlé" (Lagon, lagunes, p. 26) dans une longue scène de poème dramatique au centre de Lagon, lagunes. S'assumer comme métis, c'est d'abord en revendiquer le nom. Joël Des Rosiers, poète et essayiste haïtien installé à Montréal, "assume» le fait d'être métis et d'appartenir par sa double généalogie à l'Afrique et aux Amériques:

Je suis un métis, je suis africain, européen, amérindien. Quant à ma part africaine, selon toute vraisemblance, je pourrais être le fils d'une peuhle et d'un mandingue. Tous ces fragments font de moi un homme d'Amérique, continent de toutes les migrations. L'Amérique est l'Afrique promise. Peu d'espaces culturels dans le monde portent en eux autant de mémoires ${ }^{15}$.

Cette appartenance au "tiers-espace» de l'Amérique caractérise la situation de Sylvie Kandé, franco-sénégalaise née en métropole, et qui vit à New York, où elle enseigne depuis de longues années.

C'est précisément parce qu'il transgresse le "Code noir» de la séparation des «races», que le métissage permet de «défaire les dichotomies

I4. S. Kandé, Lagon, lagunes - tableau de mémoire, p. 15 sq.

I5. J. Des Rosiers, Théories caraïbes, poétique du déracinement, p. 188. 
et les oppositions binaires qui tiennent trop souvent lieu d'explication commode» et d'«explore[r] le poids de l'histoire sur la formation du concept ${ }^{16}$.

Ce motif du métissage, central dans Lagon, lagunes, reste présent, quoique de manière plus discrète, dans le deuxième volume de poésie, publié en 2011, La quête infinie de l'autre rive, épopée en trois chants, consacré au rêve fou de la traversée de l'Atlantique d'Afrique en Amérique par une flotte de pirogues. Dans la note introductive au volume, Sylvie Kandé adapte le récit de l'historien al-Umari sur le projet de navigation épique rapporté par l'empereur Mansa Moussa, successeur du roi Aboubakar:

Le roi qui fut mon prédécesseur, se refusant à croire qu'il était impossible de découvrir l'extrême limite de l'Océan, brûlait de le faire. Il équipa donc deux cents pirogues pour ses marins et deux cents autres remplies d'or, d'eau et de provisions en suffisance pour des années. Puis il ordonna au capitaine de ne revenir qu'après avoir atteint l'autre rive ou bien encore si l'eau ou les vivres venaient à manquer ${ }^{17}$.

Après ces préparatifs, le second chant du poème épique s'achève sur les noces organisées par Mori Mansa, roi du Mali, qui donne sa sœur Kafuma au Cacique d'Amérique. Cette union, sur laquelle «s'arrête la geste d'Aboubakar» (p. 81), accomplit les "épousailles» des deux continents, desquelles doit naître une poésie nouvelle, afro-américaine stricto sensu: "C'est ainsi que l'Afrique et l'Amérique s'épousèrent» (p. 81).

La pirogue, qui reprend en mode mineur le motif épique des vaisseaux des grandes navigations héritées d'Homère, de Virgile aussi bien que des sagas nordiques et de Camoes ou, plus récemment de Saint-John Perse dans Amers, ou de Glissant dans Les Indes, accomplit ces noces cosmiques entre les continents. Chez Kandé, la flotte si légère d'Aboubakar II trace un trait d'union charnel entre l'Afrique et l'Amérique en perpétuant la mémoire du "passage du milieu», accompagnée des vers du Cahier d'un retour au pays natal cité en exergue: "Donnez-moi les muscles de

I6. "Le poème comme geste», entretien de Sylvie Kandé avec Elara Bertho, 13 mars 2017.

17. S. Kandé, La quête infinie de l'autre rive, épopée en trois chants, p. 13. 
cette pirogue sur la mer démontée / et l'allégresse du lambie de la bonne nouvelle!». Ce récit-Relation de l'enchevêtrement se perçoit aussi dans un autre poème de l'Haïtien René Depestre, dédié à Césaire et intitulé "La pirogue de Léopold Sédar Senghor", où l'embarcation est l'emblème d'une poétique de la négritude, à laquelle Depestre a lui-même réfléchi de manière critique dans l'essai Bonjour et adieu à la négritude (1989):

Malgré tout le malheur nègre des fonds de cale, il a su pénétrer le mystère du monde blanc qui fait encore pleurer de rage nos fictions. La poésie met au bien les muscles puissants De la pirogue où Senghor et Césaire réveillent Dans nos souvenirs la chaux vive de la mer ${ }^{18}$.

La pirogue, par laquelle s'établit la médiation transatlantique des continents, est riche de connotations sexuelles, comme dans le beau poème "Congo" des Éthiopiques de Senghor: "Femme grande! eau tant ouverte à la rame et à l'étrave des pirogues / Ma Saô mon amante aux cuisses furieuses, aux longs bras de nénuphars calmes", qui se clôt sur la reprise des mêmes motifs: "Mais la pirogue renaîtra par les nénuphars de l'écume / Surnagera la douceur des bambous au matin transparent du monde» ${ }^{19}$.

La matrice du long poème de Kandé La quête infinie de l'autre rive se trouve peut-être dans le paragraphe du Cahier qui associe la pirogue à des images phalliques, dans une cosmogonie pan-érotique où sont consommées les noces de l'homme et de la mer, mais aussi de l'Afrique et des Amériques, dans une "prière virile»:

et voici par vingt fois d'un labour vigoureux la pagaie forcer l'eau

la pirogue se cabre sous l'assaut de la lame, dévie un instant, tente de fuir, mais la caresse rude de la pagaie la vire, alors elle fonce, un frémissement parcourt l'échine de la vague, la mer bave et gronde la pirogue comme un traineau file sur le sable.

I8. R. Depestre, «La pirogue de Léopold Sédar Senghor», p. 20.

19. L. S. Senghor, Poèmes, p. 101 et 103. 
Au bout de ce petit matin, ma prière virile :

Donnez-moi les muscles de cette pirogue sur la mer démontée

Et l'allégresse convaincante du lambi de la bonne nouvelle! ${ }^{20}$

Chez Sylvie Kandé, la pirogue est au contraire représentée comme un corps féminin tendrement caressé par le roi Aboubakar, toujours dans une union amoureuse:

Penché sur le plat-bord et cerné de silence

Aboubakar s'adresse à sa pirogue

flattant et cajolant quant et quant son bois

d'une main chaude qui exige et qui octroie

pénétrée par ce lent monologue

où l'impair au rêve se mêle

la rétive sent bomber sa peau ligneuse

et ses flancs s'engorger d'un semblant de sève

elle gémit et ondoie tandis que Bori murmure

se tait effleure feint la réticence

pour enfin offrir de plus savantes caresses

m’amie ma geôle mon épouse

combien que nous dussions mourir

lui souffle-t-il soucieux toutefois

d'être bien entendu de tous

il importe que grâce à toi

notre bon plaisir et unique passion

s'enflent aux dimensions d'une époque

ainsi parle à sa séditieuse barquée

Manden Mori où il n'y a que vaillance et noblesse ${ }^{21}$

\section{Élégie des gestes perdus}

En guise d'action, l'«épopée en trois chants" - selon le sous-titre donné à La quête infinie de l'autre rive - se concentre sur les gestes des rameurs qui «cadencent leurs corps / pour fendre de la pointe gâtée de l'aviron / les mottes violettes de la grande savane salée / que nul sillon ne marque / où nulle semence ne lève» (p. 17). Mais aussi, comme il se doit d'une

20. A. Césaire, Cahier d'un retour au pays natal, p. 51 sq.

2I. S. Kandé, La quête infinie de l'autre rive, épopée en trois chants, p. 59 sq. 
chronique, sur les faits et gestes du roi Aboubakar - "gestes mélodieux" (p. 19, p. 30), gestes amoureux, "d'une main chaude», «flattant et cajolant", et parfois gestes violents. La parole elle-même est présentée comme un geste, à l'instar de la "parole geste» authentique qui, pour Ramuz, s'oppose à la "parole signe» des convenances sociales:

Je dis qu'il parle peu musclant son geste

et affûtant le tranchant de son verbe - suspicieux

ô combien de la parole qui s'épanche et mime ${ }^{22}$

Le long poème La quête infinie de l'autre rive, épopée en trois chants, en ce sens, doit être considéré non pas seulement comme un «récit néoépique" (p. 14) mais, à la lettre, comme une chanson de geste, au sens du français moderne aussi bien que de l'ancien français. Il anticipe le choix formulé par Kandé en 2017 à propos du recueil Gestuaire: «j'ai voulu parler de la manière dont les gestes jalonnent un parcours de vie, gestes eux-mêmes chargés de signification historique " ${ }^{23}$. Lorsqu'elle explicite la signification qu'elle donne au beau titre Gestuaire, l'auteure se réfère à Michel Leiris pour faire des gestes l'objet même du poème:

De même que Michel Leiris, dans Glossaire, serrait ses gloses, dans Gestuaire, je serre mes gestes. Des gestes de mort et de vie, d'extrême tendresse et de violence inouie, qui fissurent la surface lisse de l'évidence et bouleversent le temps dans sa course annoncée ${ }^{24}$.

Au-delà du néologisme - «gestuaire» désigne bien un recueil de gestes -, la référence à l'écrivain et ethnologue, auteur de L'Afrique fantôme et de Contacts de civilisation aux Antilles, ami proche d'Aimé Césaire et cofondateur de Présence africaine, n'a rien d'étonnant pour des poèmes dédiés à la mémoire de l'esclavage et du marronnage. Le titre est lui-même riche de polysémie. Historienne de profession, Sylvie Kandé inscrit ces gestes du quotidien dans le traumatisme de la colonisation, de l'esclavage, de la ségrégation. Sylvie Kandé affirme qu'elle «dresse une nomenclature de gestes divers", de gestes qu'elle qualifie d' «éloquents" ${ }^{25}$.

22. Ibid., p. 46.

23. "Le poème comme geste», entretien de Sylvie Kandé avec Elara Bertho, 13 mars 2017.

24. S. Kandé, La quête infinie de l'autre rive, épopée en trois chants, p. 103.

25. "Le poème comme geste», entretien de Sylvie Kandé avec Elara Bertho, 13 mars 2017. 
Le recueil de 2016, Gestuaire, est ainsi hanté par la violence coloniale (et postcoloniale) - "gestes de mort et de vie, d'extrême tendresse et de violence inouïe» (p. 103). Les poèmes «Tous comptes faits" et "Cannes» évoquent directement la "mémoire de la Traite» (Glissant) et celle de "Morts en guerre" reprend le thème senghorien des "hosties noires" de la guerre. Ainsi, surtout, de "Génocide", poème en prose en forme d'apologue à l'ironie toute baudelairienne, qui définit le maniement du couteau comme «l'art des gestes», qui sublime le «mouvement»:

En bas, la rue aiguisait en riant ses couteaux.

- Pourquoi eux? Comment calculez-vous la différence? Que ne donnez-vous l'ordre de suspendre cette tuerie avant qu'elle ne s'étende? - C'est que nous, nous maîtrisons l'art des gestes; eux, depuis la nuit des temps, se contentent de mouvements. Advienne que pourra. Pour ma part, je m'en lave les mains.

En histrion consommé, il joignit le geste à la parole.

Éclata ce premier hurlement - un à vous glacer le sang - suivi de vociférations. Par la baie vitrée, on vit les lames se mettre à trancher, méthodiques, et quelques corps danser grotesquement avant de s'effondrer.

Ce qu'il fallait démontrer, sans doute ${ }^{26}$.

Le geste est voué par définition à disparaître. "Ombre portée du néant", il a partie liée avec les forces de la mort. Sous le signe de l'absence et du deuil, le volume exprime la «Mélancolie, ce sixième sens!» (p. 11), mélancolie élégiaque comme le montre le bouleversant poème «Brève de main»:

Car du geste qui ne s'entend ni ne s'écrit n'est-il pas juste de dire qu'il est pensée qui s'effile dans l'air propos qui cogne le vide ombre portée du néant... ${ }^{27}$

Le poème "Feu ce phalène mien", sous-titré "chant triste», "long thrène messager / qui son encre déroule / et lentement ses sanglots» (p. 64) est une élégie à l'agonie des phalènes à la lampe du soir, métaphore de la vie elle-même comme "fortitude triste» des espoirs déçus. "L'oiseau

26. S. Kandé, Gestuaire, p. 26.

27. Ibid., p. 40. 
de septembre" met en scène l'agonie d'une vieille tante qui étrangle ses canaris. Le titre Gestuaire fait discrètement allusion au vocabulaire funèbre du reliquaire et de l'ossuaire. Le lecteur peut même y entendre un écho de la première édition des poèmes après la mort de Rimbaud, publiée par Rodolphe Darzens en 1891 sous le titre Reliquaire.

Les enchevêtrements de la mémoire, du deuil et du renouveau se lisent aussi à rebours du temps dans les recueils de Sylvie Kandé, car déjà dans la "néo-épopée» consacrée à la traversée de l'Atlantique en 2011, La quête infinie de l'autre rive, l'auteure oppose le poème, "monument de mots» à la mémoire de l'expédition maritime vers les Amériques, à un "ossuaire»:
Quelle que soit l'issue
battez la vague et cavez le vent:
ce n'est pas un ossuaire
qu'aux grands fonds vous apprêtez
c'est un monument de mots
que glorieusement nous nous érigeons
afin que le récit retourne à la terre
sans jamais s'y ensevelir pour autant ${ }^{28}$

L'ossuaire, où les ossements sont dispersés et entremêlés dans une confusion obscène, porte atteinte à la dignité des défunts. Dès lors, il appartient à la poésie, et à l'art en général, de «réparer» non pas tant les vivants que les morts, et de les rétablir dans leur dignité de personnes humaines. Comme la "Pharaonne» que le "geste lapidaire» fixe dans sa "pose féconde dans un basalte coulée» (p. 94), «le geste le plus tendre est inscrit dans la pierre» (p. 95). De même que la vahiné de Gauguin devient «immortelle par [1]a main» (p. 62) du peintre, les rameurs des pirogues d'Aboubakar sont immortalisés par le poème. L'épopée en trois chants est bien en ce sens un "tombeau" qui donne forme et sens à la mort des héros par une construction de langage. Par ricochet analogique, Gestuaire «invit[e] les morts au bal» (p. 18), car la poésie a pour fonction de perpétuer les gestes, afin qu'ils ne soient pas ensevelis de manière anonyme dans la fosse commune du temps perdu. "Bal de pierre», par exemple, représente ce travail d'édification du poème comme tombeau

28. S. Kandé, La quête infinie de l'autre rive, épopée en trois chants, p. 38. 
en évoquant la figure du père disparu, qui "se retourne dans sa tombe" et «se recouche à la manière de ceux des limbes / rabattant sur le dédain de son épaule / une lourde courtepointe de terre et de gazon» (p. 19).

Le modèle de l'image, de la photographie, ou de la peinture - celle d'Édouard Duval-Carrié, par exemple, ou d'Alice Martinez-Richter -, s'impose en ce que le cours du temps s'y trouve suspendu. Les poèmes de Gestuaire prennent ainsi volontiers la forme de l'ekphrasis, comme "Cannes", inspiré du tableau "Adam et Ėve» d'Alice Martinez-Richter de 1949, ou encore «Survivre selon Alice», d'après un tableau de 1974:

Dans ce pays sans merveille

dans ce paysage à penser

jamais à la journée ne suffit sa peine

mais à moisson si vaine esprit plus fécond ${ }^{29}$

Le travail du peintre avec son chevalet est directement représenté, sous le signe de la main: "Son geste s'arrondit pour décrire leur séquelle" (p. 25). Fixé par l'image, le geste reste en suspens, hors du temps - le mouvement devient danse. Ainsi, à sa manière, à rebours d'une modalité épique, Gestuaire présente un "monument de mots", une élégie à la gloire des gestes perdus - non pas ceux héroïques de la "chanson de geste» ou de la saga de La quête infinie de l'autre rive, mais bien des gestes quotidiens et infinitésimaux des "vies minuscules", des vies ordinaires, comme ceux du père, auquel le recueil rend hommage:

de ses mains j'ai fait mon deuil

mais de ses gestes non

une façon de suspendre au clou sa canadienne

et de rêver bref en surplomb de son soulier $[\ldots]^{30}$

La tonalité élégiaque de "L'oiseau de septembre» est proche de l'univers des poèmes de Pierre Reverdy, lorsque ceux-ci tentent de soupeser «le poids de l'air ${ }^{31}$ :

Gestes au miroir dans une masure sans rime ni raison

Une porte grince une persienne claque - conversations

29. S. Kandé, Gestuaire, p. 24.

3o. Ibid., p. 34.

3I. P. Reverdy, Sources du vent, p. 183. 
Ce n'est rien juste le temps qui tente de battre la mesure

Arpente ses quartiers vétustes en claquant du talon

Du vestibule à la souillarde du cellier à la soupente

La clé la clé est dans la redondance le reflet le renom ${ }^{32}$

Après avoir rencontré le chorégraphe Jean-Claude Gallotta à Grenoble, en 1984, Hervé Guibert demandait: "Qui est le chorégraphe sinon ce grand fada sacré que la société semble payer pour le rachat de la mort des gestes?» En 2012, dans un spectacle magnifique intitulé Racheter la mort des gestes, Gallotta a affronté le défi de suspendre le mouvement et de perpétuer l'éphémère. Rivalisant avec la danse, mais aussi la photographie et la peinture, Sylvie Kandé assigne ainsi, à son tour, à la poésie la tâche de "Racheter la mort des gestes" dans une épopée intime du quotidien, en mode mineur.

\author{
Dominique Combe \\ École normale supérieure, \\ Paris Sciences Lettres
}

32. S. Kandé, Gestuaire, p. 20. 


\section{BIBLIOGRAPHIE}

Born in slavery: slave narratives from the federal writers' project, 19361938, Library of Congress, Washington D.C., en ligne <https:// www.loc.gov/collections/slave-narratives-from-the-federal-writersproject-1936-to-1938/about-this-collection/>.

CÉsaire, Aimé, Moi, laminaire, Paris, Seuil, 1982.

—, Cahier d'un retour au pays natal, Paris, Présence africaine, 1983.

Depestre, René, "La pirogue de Léopold Sédar Senghor", in Nonassistance à poètes en danger, Paris, Seghers, 2005, p. 20.

Des Rosiers, Joël, Théories caraïbes, poétique du déracinement, Montréal, Triptyque, 2009.

Glissant, Édouard, "Aux orées des archipels», postface à Sylvie Kandé, Lagon, lagunes - tableau de mémoire, Paris, Gallimard, 2000, p. 75-76.

Kandé, Sylvie, Terres, urbanisme et architecture "créoles» en Sierra Leone, XVIII ${ }^{e}$-XIX ${ }^{e}$ siècles, Paris, L'Harmattan, 1998.

—, Discours sur le métissage, identités métisses. En quête d'Ariel, Paris, L'Harmattan, 1999.

—, Lagon, lagunes - tableau de mémoire, Paris, Gallimard, 2000.

—, La quête infinie de l'autre rive, épopée en trois chants, Paris, Gallimard, 2011.

—, Gestuaire, Paris, Gallimard, 2016.

—, "Le poème comme geste», entretien avec Elara Bertho, Diacritik, 13 mars 2017, en ligne <https://diacritik.com/2017/03/13/entretien-avec-sylvie-kande-le-poeme-comme-geste/>.

Reverdy, Pierre, Sources du vent, Paris, Poésie/Gallimard, 1971 (1929).

Senghor, Léopold Sedar, Poèmes, Paris, Seuil, 1964. 